\title{
Magnetic Properties of SMC Cores Produced at a Low Compacting Temperature
}

\author{
A. Jakubas ${ }^{a *}$, P. GęBara ${ }^{a}$, S. Seme ${ }^{b}$, A. Gnatowski ${ }^{a}$ And K. Chwastek ${ }^{a}$ \\ ${ }^{a}$ Częstochowa University of Technology, H. Dąbrowskiego 69, 42-201 Częstochowa, Poland \\ ${ }^{b}$ University of Maribor, Hočevarjev trg 1, 8270 Krško, Slovenia
}

\begin{abstract}
Soft magnetic composites are readily used in the design of electromagnetic converters and electrical machines. In the paper, the magnetic properties of "home-made" cores made of iron powder and suspense polyvinyl chloride are examined. Samples were prepared by hot pressing of a PVC/Fe powder mixture with a different ratio of constituent components. Phase composition was investigated using an X-ray diffractometer and the presence of $\alpha$-Fe was detected. Measurements of magnetic permeability revealed its allometric decrease with an increase of PVC content in composite. For the description of magnetization curves the phenomenological Jiles-Atherton model is used. The results of simulations are in a qualitative agreement with experiment.
\end{abstract}

DOI: 10.12693 /APhysPolA.131.1289

PACS/topics: 75.60.-d, 75.60.Ej, 77.84.Lf

\section{Introduction}

Soft magnetic composites (SMCs) have recently gained the attention of the scientific community $[1,2]$. These materials reveal interesting physical and chemical properties, which may be easily fine-tuned to specific applications by an appropriate material processing, which can be schematically depicted with the diagram below (Fig. 1).

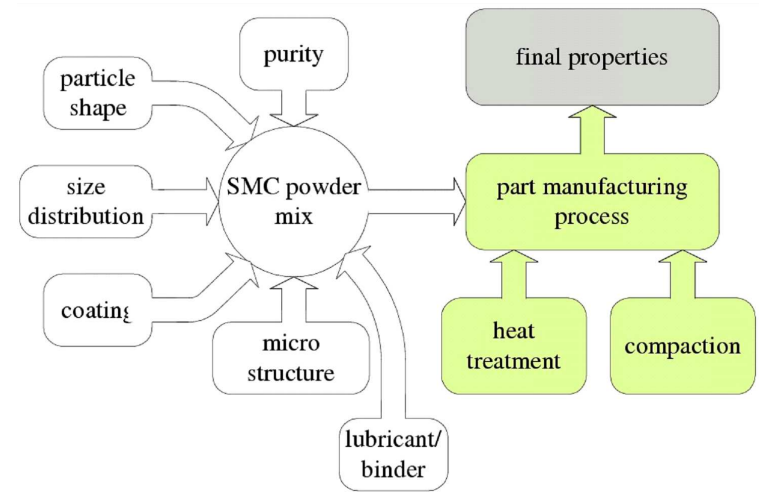

Fig. 1. Factors affecting properties of magnetic circuits made of SMCs.

Gilbert et al. examined the effects of processing variables and methods on density, structure and magnetic properties for two commercially available SMC materials for use in low loss applications [3]. Shokrollahi and Janghorban focused on the effects of compaction parameters and particle size [4] and different annealing treatments [5] on magnetic properties of iron-based alloys. Anhalt carried out a systematic study of the dependences of coercive field strength, maximum permeability and dynamic losses

*corresponding author; e-mail: jakubasa@el.pcz.czest.pl on particle size [6]. Ding et al. fabricated a number of $\mathrm{Fe} /$ silicate glass SMCs differing in particle size and volume fraction of iron and examined the variations of density, electrical, thermal and magnetic properties (maximum permeability, coercivity, residual magnetic flux density, core loss) of the developed cores [7]. Kollár et al. [8] and Lauda et al. [9] focused on the study of dynamic magnetic properties of Fe-based composites in a wide range of excitation frequencies. Ślusarek et al. examined a correlation between SMC density and magnetic properties of ready-made cores [10].

The engineering community has noticed the advantages resulting from the application of powder metallurgy in the design of electromagnetic converters and electric machines [11-15]. Insulated iron powder (compacted and heat treated) offers an attractive alternative to conventionally used steel laminations in components requiring soft magnetic materials. Magnetic circuits made of SMCs may be precisely machined, which limits the necessity to perform additional mechanical post-processing e.g. tooling of parts [16]. Their production costs are significantly lower than in the case of conventional materials, moreover the recycling of worn-out components is significantly simpler. The SMCs allow one to design low-dimensional magnetic circuits with complicated geometries. An important feature of SMCs is the isotropy of their magnetic properties, which results in the possibility of developing electrical machines with 3D magnetic fields with a reduction of production costs at the same time.

Most research work carried out on SMCs is based on commercially available products like Somaloy from Höganäs $[3,10,17]$. For these products the processing parameters are relatively high, e.g. the curing temperature is of the order of $500^{\circ} \mathrm{C}$, whereas the compacting pressure is of the order $800 \mathrm{MPa}$. In this communication, we report on the possibility to manufacture "home-made" SMC cores using readily available components. For our SMCs the processing parameters were significantly lower: 
the curing temperature $165^{\circ} \mathrm{C}$ and the pressure approximately $200 \mathrm{MPa}$. A similar approach has been considered recently by Ferraris et al. [18].

The properties of "home-made" SMCs shall never be as good as those for high quality brand products. Instead, it is expected that the developed materials may have properties comparable to MnZn ferrites [19] and thus they may be used in similar applications (e.g. EMI suppression filters, chokes, small-size impedance matching transformers). It should be stressed that for the production of "home-made" SMCs waste materials might be used, which is an eco-friendly solution in accordance with worldwide trends, cf. [20].

\section{Material and methods}

Several samples have been prepared from iron $(99 \%$ pure Fe, granulation 100-150 $\mathrm{mm}$ ) powder and suspense polyvinyl chloride PVC-S (granulation 15-100 $\mu \mathrm{m}$ ). Fine structure of both components allowed us to obtain highly homogeneous mixtures. Polyvinyl chloride used as the matrix has good mechanical properties. Moreover, it is resistant against the action of many solvents and is a good electrical insulator. From the obtained mixtures, cylinder-shaped samples were formed using a hydraulic press with a form and a band with a thermocouple for controlling heat treatment conditions, cf. Fig. 2. The dimensions of all samples were: the outer radius $-25 \mathrm{~mm}$, the inner radius $-15 \mathrm{~mm}$ and height $-10 \mathrm{~mm}$. The same number of coils was used for all cores: the primary winding -100 coils, the secondary one -30 coils. The weight of the samples was about $75 \mathrm{~g}$.

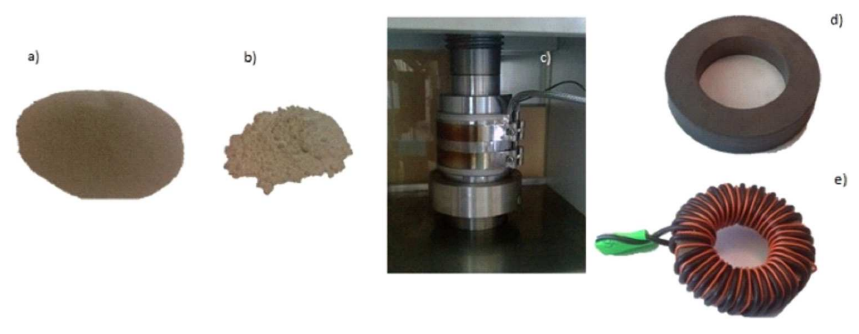

Fig. 2. The material under study at different stages of preparation: a) raw Fe powder, b) PVC-S, c) the hydraulic press with a heating band, d) the prepared core, e) the core with windings.

The first stage of research was the determination of optimal PVC content to make up stable samples, able to maintain reasonably good magnetic properties. The tests were started at $20 \mathrm{wt} \%$ polymer content, yet for further studies four samples with appropriately higher Fe content were chosen. The raw materials were mixed using weight proportions. The PVC contents in the final samples were equal to $0.5,1.0,1.5$, and $2.0 \%$, respectively. The cylinder-shaped samples were next subject to reticulation for $30 \mathrm{~min}$ at $165^{\circ} \mathrm{C}$ and at pressure of $200 \mathrm{MPa}$.

The composition of ready-made samples was verified using an X-ray diffractometer Bruker D8 Advance with $\mathrm{Cu} K_{\alpha}$ radiation and LynxEye detector. An exemplary
XRD pattern is shown in Fig. 3. It can be stated that the microstructure is highly homogeneous. Diffraction reflexes observed in the pattern are typical for $\alpha$-Fe.

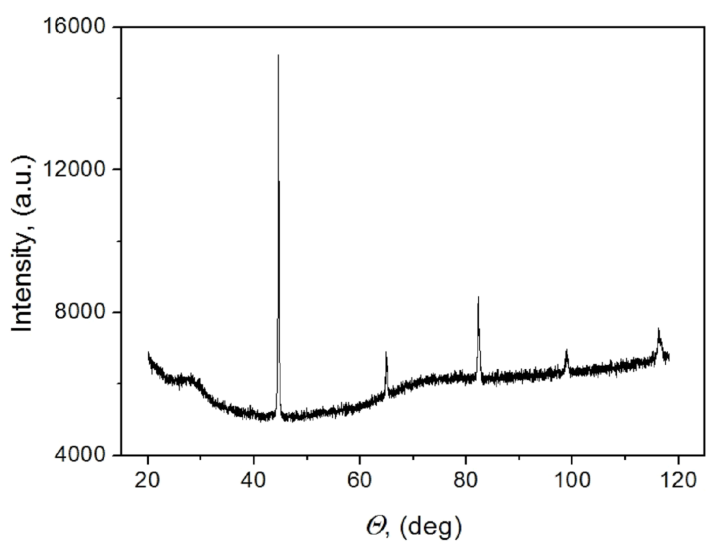

Fig. 3. An exemplary XRD pattern collected for the developed cores.

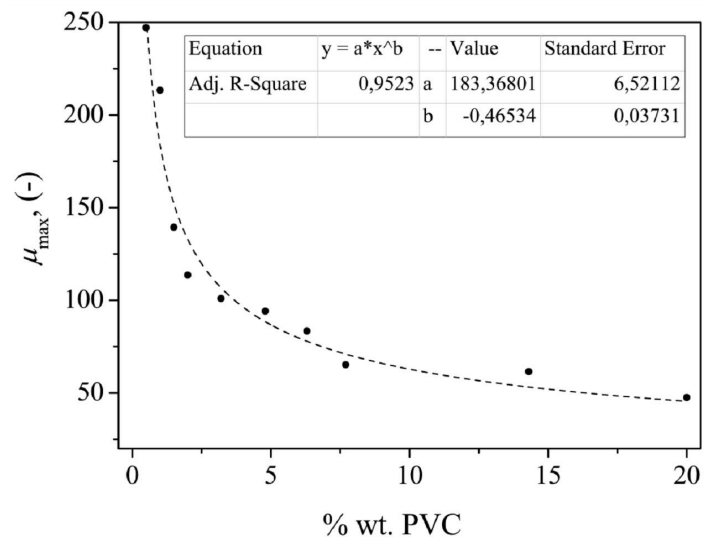

Fig. 4. Maximum permeability vs. PVC content in the examined cores.

Magnetic properties were determined using a computer-aided measurement setup. In Fig. 4, the dependence of maximum permeability on polymer content measured at $B_{m}=0.4 \mathrm{~T}, f=50 \mathrm{~Hz}$ is presented.

In order to describe the measured hysteresis loops, the phenomenological model advanced by Jiles and Atherton was used [21]. This hysteresis model has attracted the attention of the scientific community in the last three decades due to the uncomplicated structure of its equations, which may be easily solved with contemporary computers. Important advantages are the physical interpretation attributed to its parameters and the possibility to incorporate different physical phenomena in the description within the framework of "an effective field". Despite some of its weaker points (non-closure of minor loops for complicated magnetization patterns and the necessity to "patch" model equations in order to describe 
material behavior after a field reversal [22-24], this description remains one of the most commonly used hysteresis models in the practice.

The Jiles-Atherton (JA) model was applied previously for the description of hysteresis curves in SMC materials by Benabou et al. [25] and by Miljavec and Zidarič [23].

\section{The Jiles-Atherton model}

The Jiles-Atherton (JA) model is based on the assumption that hysteresis occurs as the result of energy dissipation on imperfections existing in the material (voids, inclusions, deformations of crystalline lattice etc.), termed as pinning sites during domain wall movement. The basic set of model equations used in this paper is as follows:

$$
\begin{aligned}
& \frac{\mathrm{d} M}{\mathrm{~d} H_{\mathrm{eff}}}=\frac{\delta_{M}\left(M_{a n}-M\right)}{k \delta}, \\
& M_{a n}=M_{s}\left[\operatorname{coth} \frac{H_{\mathrm{eff}}}{a}-\frac{a}{H_{\mathrm{eff}}}\right], \\
& H_{\mathrm{eff}}=H+\alpha M .
\end{aligned}
$$

There are four model parameters: $M_{s}[\mathrm{~A} / \mathrm{m}]$ (saturation magnetization), a $[\mathrm{A} / \mathrm{m}]$, the shape parameter in the modified Langevin function (2), which describes the so-called "anhysteretic" magnetization $M_{a n}, \alpha$, a dimensionless constant, which describes mutual interactions between magnetic domains within the material, and $k$, $\mathrm{A} / \mathrm{m}$, which is proportional to the product of density of pinning sites and their average energy. This quantity may be assumed as approximately proportional to coercive field strength measured in quasi-static conditions. The sign of $\mathrm{d} H / \mathrm{d} t$ is denoted with $\delta= \pm 1$. It is introduced in order to distinguish the ascending and the descending branch of a hysteresis loop. The pseudo-parameter $\delta_{M}$ is introduced in order to avoid negative $\mathrm{d} H / \mathrm{d} t$ slopes after a sudden field reversal, $\delta_{M}=$ $0.5\left[1+\operatorname{sgn}\left(\left(M_{a n}-M\right) \mathrm{d} H / \mathrm{d} t\right)\right]$.

The set of Eqs. (1)-(3) may be transformed into an explicit relationship for differential susceptibility $\mathrm{d} M / \mathrm{d} H$ and given in the following form:

$$
\frac{\mathrm{d} M}{\mathrm{~d} H}=\frac{\delta_{M}\left(M_{a n}-M\right)}{k \delta-\alpha \delta_{M}\left(M_{a n}-M\right)} .
$$

Model equations used in this paper are simplified in comparison with those considered in the paper [24], as the separation of total magnetization into irreversible and reversible components is not considered. As pointed out by Zirka et al. [24], the possibility to carry out such separation is arguable. The reversibility parameter $c$, introduced by Jiles and Atherton [26], has to be updated for different excitation levels [23].

In order to capture the effect of varying polymer weight content in modeling, two approaches are tested:

1. the shape parameter $a$ of the "anhysteretic" curve is made dependent on the relative content of the iron phase,

2 . the $\alpha$ parameter, which accounts for mutual interactions within material, is modified accordingly. The latter possibility may be qualitatively explained by consideration of inner demagnetization fields [27].

As far as the first possibility is concerned, the inspection of experimental data chosen for analyzing the trends in the shapes of modeled hysteresis loops led us to the conclusion that a sudden decrease of average permeabilities was observed for the measured hysteresis loops for PVC volume fractions over $15 \%$. Therefore we decided to consider some nonlinear dependences for the dependence of $a$ parameter on iron weight content. In the first attempt, we tried to apply a nonlinear weighting scheme similar to the general logarithmic equation considered by Anatharaman et al. for dielectric constants in rubber ferrite composites [28]:

$$
\begin{aligned}
& \log a=-\sum_{i} w_{i} \log a_{i}= \\
& -w \log a_{F E}-(1-w) \log a_{P O L} \approx-w \log a_{F E} .
\end{aligned}
$$

Thus the value of parameter $a$ was assumed to decay exponentially upon the increase of iron volume fraction, $a=a_{0} \exp (-w)$. The minus sign before the summation may be explained by noticing the duality or counterpart roles played either by electric permittivities and magnetic permeabilities or capacitances and inductances in general [29].

During preliminary tests it turned out that the generalized exponential function in the form $a=A a_{0}^{-w}$ offered better flexibility in the description.

In the second approach it was assumed that the value of the parameter responsible for mutual interactions between magnetic domains in the material ( $\alpha$ in the JA model) varied for different Fe volume fractions. As the iron content decreases, the demagnetization effects tend to play a more significant role, thus the effective $\alpha$ value experienced by the domains is decreased. For "almost pure" Fe samples, the hysteresis loops are the steepest and $\alpha$ approaches its maximal, critical value. We assumed that the variation of $\alpha$ value might also be described with the function

$$
\alpha=C \alpha_{0}^{w} \text {. }
$$

\section{Modeling}

Measurements of hysteresis curves were carried out using a computer-aided setup for four cores with PVC wt\% contents: $0.5,1.0,1.5$, and $2.0 \%$. Exemplary results are depicted in Fig. 5. Upon the increase of polymer content, the hysteresis curves became more flattened. The excitation frequency was so chosen that the modification of loop shapes e.g. due to the flow of inter-grain eddy currents could be neglected.

The set of model parameters was estimated using the robust "branch-and-bound" method [30, 31] for one of the loops. In the subsequent modeling the values of all parameters apart from $a$ (case 1 ) or $\alpha$ (case 2) were kept constant. Figure 6 depicts the modeling, results for case 1 (varying the value of parameter $a$ ), whereas Fig. 7 - for case 2 (varying the value of parameter 


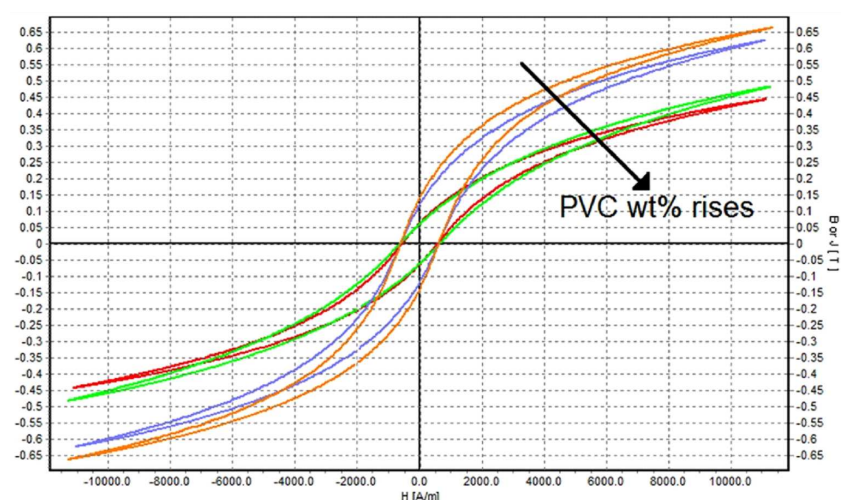

Fig. 5. An exemplary family of hysteresis loops obtained for cores with different PVC wt.\% content.

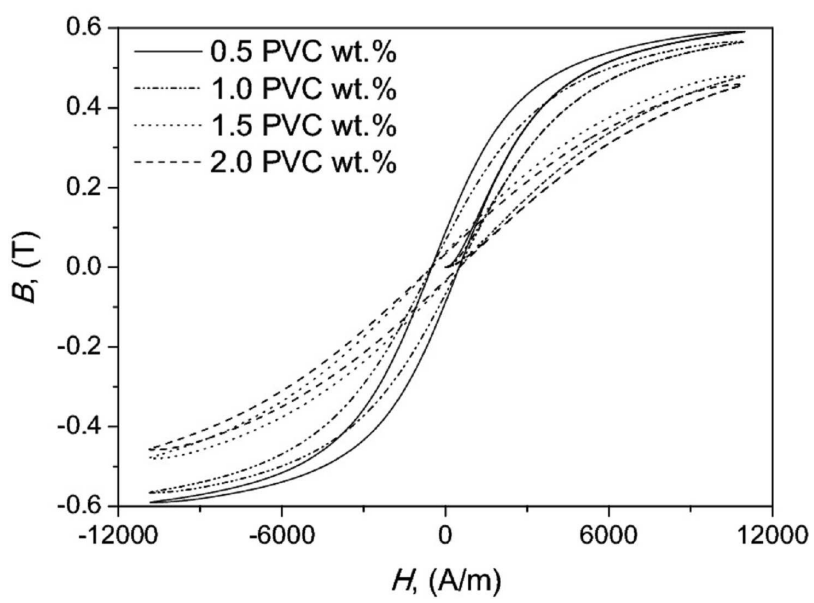

Fig. 6. The family of modeled hysteresis curves corresponding to those depicted in Fig. 5 (case 1).

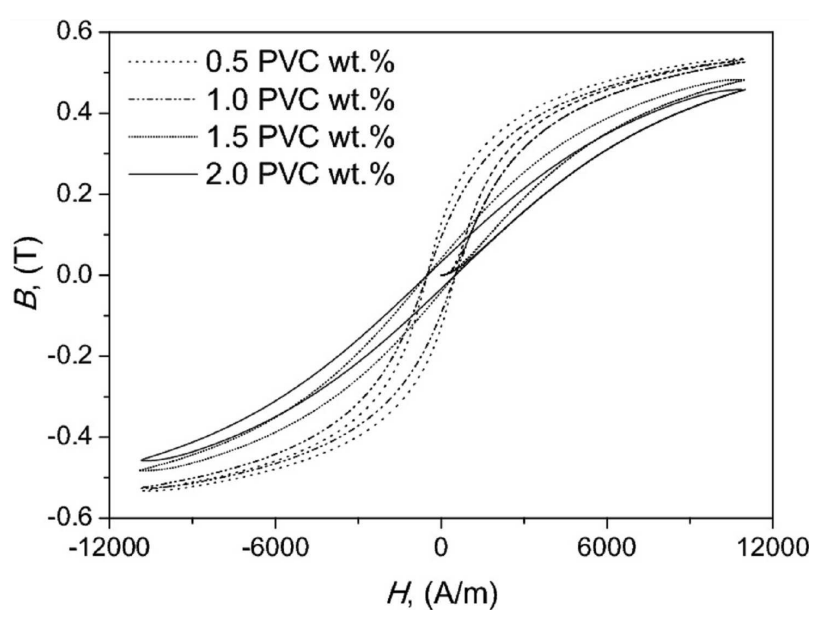

Fig. 7. The family of modeled hysteresis curves corresponding to those depicted in Fig. 5 (case 2). $\alpha)$. The dependences used for updating model parameters were $a=7.63 \times 10^{5} \times 822^{-w}$ (case 1) and $\alpha=$ $2.67 \times 10^{-7} \times\left(1.13 \times 10^{5}\right)^{w}($ case 2$)$.

From a comparison of Figs. (6) and (7), it follows that the shapes of modeled loops in both cases are in qualitative agreement with the experimental ones. However an inspection of the values for uncertainties of $a_{0}$ and $\alpha_{0}$, obtained after fitting the results to the generalized exponential laws, led us to the conclusion that the second case (introduction of functional $\alpha(w)$ dependence) might be more justified.

In order to gain some insight on the quantitative agreement of both models with the experiment, we have calculated the differences between the measured values and those predicted for the remanence points (crossing of $H$ axis). The "errors" were defined in accordance to the general formula $\varepsilon_{\%}=100 \times\left|B_{\text {rmeas }}-B_{\text {rmodel }}\right| / B_{\text {rmeas }}$.

Both models underestimated the values of remanence induction, but the second model, in which the value of effective field parameter $\alpha$ was updated, yielded a better agreement with experiment. The percentage error for the modeled remanence induction was equal to $41 \%$ (case 1 ) and $27 \%$ (case 2). These values are averaged ones for four considered loops. The relatively high values of errors may be attributed to the averaging properties of the considered JA description as well as to quite low values of the remanence induction (of the order of $0.1 \mathrm{~T}$ ), this quantity entered the denominator of the expression for $\varepsilon_{\%}$.

\section{Conclusions}

We have developed a number of cylinder shaped SMC cores differing in Fe wt\% content using readily available components. For the developed cores we have determined their basic magnetic properties and carried out modeling of hysteresis loops. For this purpose we have applied the phenomenological description advanced by Jiles and Atherton. We have found out that in order to capture the effect of varying polymer/iron ratio, it was necessary to update some values of model parameters. We have considered two approaches to address this problem. We have found out that the method, in which the value of effective field parameter $\alpha$ is made dependent on iron content, provides an improved description of the magnetization phenomena in the considered SMC cores.

\section{References}

[1] H. Shokrollahi, K. Janghorban, J. Mater. Process. Technol. 189, 1 (2007).

[2] M. Najgebauer, J. Szczygłowski, Sci. J. Silesian Univ. Techn. Series Electr. Eng. 4, (220) 29 (2011) (in Polish).

[3] I. Gilbert, S. Bull, T. Evans, A. Jack, D. Stephenson, A. de Sa, J. Mater. Sci. 39, 457 (2004).

[4] H. Shokrollahi, K. Janghorban, Mater. Sci. Eng. B 134, 41 (2006).

[5] H. Shokrollahi, K. Janghorban, J. Magn. Magn. Mater. 317, 61 (2007). 
[6] M. Anhalt, J. Magn. Magn. Mater. 320, e366 (2008).

[7] W. Ding, R. Wu, Z. Xiu, G. Chen, J. Song, Y. Liao, G. Wu, J. Supercond. Nov. Mater. 27, 435 (2014).

[8] P. Kollár, Z. Birčáková, J. Füzer, J. Füzerová, R. Bureš, M. Fáberová, Acta Phys. Pol. A 118, 759 (2010).

[9] M. Lauda, J. Füzer, J. Füzerová, P. Kollár, M. Strečková, M. Fáberová, Acta Phys. Pol. A 126, 144 (2014).

[10] B. Ślusarek, J. Szczygłowski, K. Chwastek, B. Jankowski, COMPEL 34, 1 (2015).

[11] A. Hamler, V. Goričan, B. Šuštaršič, A. Sirc, J. Magn. Magn. Mater. 304, e812 (2006).

[12] C. Cyr, Ph.D. Thesis, Université Laval, Québec, 2007.

[13] G. Cvetkovski, L. Petkovska, IEEE Trans. Magn. 44, 3812 (2008).

[14] K. Vijayakumar, R. Karthikeyan, G.K. Sathishkumar, R. Arumugam, in: Proc. TENCON'2008, Hyderabad (India) 2008.

[15] R.M. Wojciechowski, C. Jędryczka, P. Łukaszewicz, D. Kapelski, COMPEL 31, 1528 (2012).

[16] Höganäs AB, Sweden.

[17] L.L. Evangelista, D.S. Avila, M.A. Carvalho, H.D. Lopes, IEEE Trans. Magn. 52, (5) 1 (2016).

[18] L. Ferraris, E. Pošković, F. Franchini, AIP Adv. 6 056209 (2016)
[19] A. Goldman, Modern Ferrite Technology, Springer, New York 2006.

[20] L. Zhang, Z. Xu, J. Cleaner Prod. 127, 19 (2016).

[21] D.C. Jiles, D.L. Atherton, IEEE Trans. Magn. 19, 2183 (1983).

[22] A. Benabou, J.V. Leite, S. Clénet, C. Simăo, N. Sadowski, J. Magn. Magn. Mater. 320, e1034 (2008).

[23] D. Miljavec, B. Zidarič, J. Magn. Magn. Mater. 320 , 763 (2008).

[24] S.E. Zirka, Yu.I. Moroz, R.G. Harrison, K. Chwastek, J. Appl. Phys. 112, 043916 (2012).

[25] A. Benabou, S. Clénet, F. Piriou, J. Magn. Magn. Magn. 261, 139 (2003).

[26] D.C. Jiles, D.L. Atherton, J. Magn. Magn. Mater. 61, 48 (1986).

[27] M. Anhalt, B. Weidenfeller, J.-L. Mattei, J. Magn. Magn. Mater. 320, e844 (2008).

[28] M.R. Anantharaman, K.A. Malini, S. Sindhu, E.M. Mohamed, S.K. Date, S.D. Kulkarni, P.A. Joy, P. Kurian, Bull. Mater. Sci. 24, 623 (2001).

[29] E.C. Cherry, Proc. Phys. Soc. 62, 101 (1949).

[30] D. Finkel, Ph.D. Thesis, North Carolina State University, Raleigh, USA 2005.

[31] K. Chwastek, J. Szczygłowski, J. Magn. Magn. Mater. 314, 47 (2007). 